\title{
CORRIGENDUM
}

\section{Nutritional access routes following oesophagectomy-a systematic review}

GA Markides, B Alkhaffaf and J Vickers

European Journal of Clinical Nutrition (2011) 65, 984; doi:10.1038/ejcn.2011.95

Correction to: European Journal of Clinical Nutrition (2011) 65, 565-573; doi:10.1038/ejcn.2010.292

Since the publication of this paper, the authors have noticed that one of the author names was incorrectly listed as B Al-Khaffaf. The correct name is shown above. 